\title{
Numerical approximation of the fractional HIV model using the meshless local Petrov-Galerkin method
}

\author{
Kunwithree Phramrung ${ }^{1}$, Anirut Luadsong ${ }^{1,2^{*}}$ (1) and Nitima Aschariyaphotha ${ }^{2}$
}

\section{"Correspondence:}

anirut.lua@kmutt.ac.th

'Department of Mathematics,

Faculty of Science, King Mongkut's

University of Technology Thonburi (KMUTT), Bangkok, Thailand

${ }^{2}$ Ratchaburi Learning Park, King Mongkut's University of Technology Thonburi (KMUTT), Ratchaburi, Thailand

\begin{abstract}
This paper deals with the model of fractional HIV-1 infection of CD4 ${ }^{+} \mathrm{T}$ cells transformation with homogeneous Neumann boundary conditions. Numerical methods for solving fractional time differential equations are developed with Caputo's definition. The forward difference methods were constructed applied to the approximation of the fractional time differential equation. The MLPG method is used to solve the problem of fractional HIV models for spatial discretization. Approximated solutions at the time level $n$ use conventional iterative methods such as fixed point iterations to handle the nonlinear parts. An analysis of stability and convergence of numerical schemes is presented along with the eigenvalue of the matrix. The abilities of the developed formula was confirmed through four numerical examples base on convergence and accuracy of numerical results. The results of the numerical experiments were compared with the solution of the integer order differential equation to confirm the accuracy and efficiency of the proposed scheme. The simulation results show that the formula is easy to use and useful for those interested in fractional derivatives.
\end{abstract}

Keywords: Caputo fractional derivative; Fractional order differential equation; HIV model; Meshless local Petrov-Galerkin method

\section{Introduction}

There are currently many countries where people are infected by the human immunodeficiency virus (HIV). Around the world, more people die from this virus. HIV is a retrovirus virus that targets $\mathrm{CD} 4^{+} \mathrm{T}$ white blood cells, the most abundant white blood cells in the immune system. Although HIV is infected in other cells as well, it can cause the most damage in $\mathrm{CD} 4^{+} \mathrm{T}$ cells by causing the cells to deteriorate and be destroyed, reducing the resistance of the immune system. Mathematical modeling is a very important branch of applied mathematics. By using this approach, we can convert problems in the real world into mathematical modules and then analyze in a better manner. Mathematical models have been proven to be valuable in understanding the dynamics of HIV infection. In 1989, Perelson developed a simple model for primary infection with HIV. This model was important in the creation of mathematical models of HIV infection. Perelson et al. expanded the model in 1993. They determined four categories: $\mathrm{CD} 4^{+} \mathrm{T}$ cells that are non-infected, $\mathrm{CD} 4^{+} \mathrm{T}$ cells that are infected, $\mathrm{CD} 4^{+} \mathrm{T}$ cells that are effectively infected and virus popu-

(c) The Author(s) 2019. This article is distributed under the terms of the Creative Commons Attribution 4.0 International License (http://creativecommons.org/licenses/by/4.0/), which permits unrestricted use, distribution, and reproduction in any medium, provided you give appropriate credit to the original author(s) and the source, provide a link to the Creative Commons license, and indicate if changes were made. 
lations. Next, Rong et al. modified the model further by incorporating anti-virus effects to study the evolution of drug resistance. They considered three classes of $\mathrm{CD} 4^{+} \mathrm{T}$ cells: non-infected cells, cells infected with the eclipse phase and effectively infected cells [1]. In Wang et al., the densities of non-infected cells, infected cells, and free viruses are assumed to be located at position $x$ at time $t$, denoted by $u(x, t), v(x, t), w(x, t)$, respectively. The spatial domain is assumed to be one-dimensional, $(\mathbf{x}, t) \in(-\infty, \infty) \times(0, \infty)$. Brauner et al. extended their work to a two-dimensional square domain $(0, l) \times(0, l)$ with periodic boundary conditions and provided a recruitment rate that was space dependent. They proposed a zero-flux boundary condition in a general bounded domain $\Omega \in \mathbb{R}^{d}$ with smooth boundary $\partial \Omega$ (homogeneous Neumann boundary condition). In recent work, Wang et al. argued that there should be a real spatial space, but generally not a square under the appropriate boundary conditions. They offered zero-flux boundary conditions in the general domain $\Omega \in \mathbb{R}^{d}$ with a smooth boundary condition. Recent studies have shown that the effectiveness of viral infection is important, indicating that the virus is transmitted to non-infected target cells. In this case, the virus particles can be transferred from infected target cells to non-infected ones through virus synapses. The results of this study suggest that direct cell-mediated infection affects the mechanism of HIV transmission through the body. Therefore, the details of cell change between HIV cells with delay and without delay can be rewritten as follows:

$$
\begin{aligned}
& \frac{\partial p(\mathbf{x}, t)}{\partial t}=\lambda(\mathbf{x})-\beta_{1}(\mathbf{x}) p(\mathbf{x}, t) r(\mathbf{x}, t)-\beta_{2}(\mathbf{x}) p(\mathbf{x}, t) q(\mathbf{x}, t)-a(\mathbf{x}) p(\mathbf{x}, t), \\
& \frac{\partial q(\mathbf{x}, t)}{\partial t}=\beta_{1}(\mathbf{x}) p(\mathbf{x}, t) r(\mathbf{x}, t)+\beta_{2}(\mathbf{x}) p(\mathbf{x}, t) q(\mathbf{x}, t)-b(\mathbf{x}) q(\mathbf{x}, t), \\
& \frac{\partial r(\mathbf{x}, t)}{\partial t}=\mathcal{D} \Delta r(\mathbf{x}, t)+k(\mathbf{x}) q(\mathbf{x}, t)-m(\mathbf{x}) r(\mathbf{x}, t)
\end{aligned}
$$

for $(\mathbf{x}, t) \in \Omega \times(0, \infty) ; \Omega \in \mathbb{R}^{d}, d=1,2,3$ with the homogeneous Neumann boundary condition $\frac{\partial r(\mathbf{x}, t)}{\partial \mathbf{n}}=0 ; \mathbf{x} \in \partial \Omega, t>0$ where $\mathbf{n}$ denotes an outward unit normal to $\partial \Omega$ and initial conditions $p(\mathbf{x}, 0)=p^{0}(\mathbf{x}) \geq 0, q(\mathbf{x}, 0)=q^{0}(\mathbf{x}) \geq 0, r(\mathbf{x}, 0)=r^{0}(\mathbf{x}) \geq 0 ; \mathbf{x} \in \Omega$. The density of non-infected cells, the density of infected cells and the density of free viruses are denoted by $p(\mathbf{x}, t), q(\mathbf{x}, t)$ and $r(\mathbf{x}, t)$, respectively. $\lambda(\mathbf{x}), a(\mathbf{x})$ and $b(\mathbf{x})$ denoted the number of newly produced non-infected cells, the death rate of non-infected cells and the death rate of infected cells, respectively. The death rate of free viruses, the transmission coefficient for virus to cell infection and the transmission coefficient for cell to cell infection are denoted by $m(\mathbf{x}), \beta_{1}(\mathbf{x})$ and $\beta_{2}(\mathbf{x})$, respectively. $k(\mathbf{x}), \mathcal{D}$ and $\Delta$ denote the rate of virus production due to the lysis of infected cells, the diffusion coefficient and the Laplacian, respectively [2].

In applied mathematics, one of the most often used concepts is that of the derivative. Derivatives show the rate of change of functions that are useful in explaining many real phenomena. The concept of fractional calculus is especially important for modeling real-world problems. Many physical problems have been simulated using the concept of fractional order derivatives. However, most fractional order differential equations that describe real-world problems are highly complex and sometimes cannot be managed through analytical methods. Therefore it is necessary to find new numerical methods for fractional order differential equations. Due to these problems, there are initial conditions, boundary condition and source terms which are difficult to find from analytical solutions. 
This means that the next state of the system does not depend on the current state only but still depends on all historical state. This is more realistic and is one reason why fractional calculus becomes more popular.

The meshless local Petrov-Galerkin (MLPG) method is a fundamental base for the derivation of many meshless formulations since the trial and test functions can be chosen from different functional spaces. The MLPG method has been proposed by Atluri and Zhu [3, 4] and Atluri, Kim and Cho [5] for solving linear and nonlinear boundary problems. This method is truly meshless, as no finite element or boundary element methods are required in this approach, either for purposes of interpolation of the trial and test functions for the solution variables or for the purpose of the integration of the weak form; while other meshless methods require background cells. Remarkable successes of the MLPG method have been reported in solving the convection-diffusion problems by Lin and Atluri [6], fracture mechanics problems by Kim and Atluri [7] and Ching and Batra [8], Navier-Stokes flows by Lin and Atluri [9], and plate bending problems by Gu and Liu [10] and Long and Atluri [11]. Based on the MLPG methods concept, these variants of the MLPG method are labeled MLPG1, MLPG2, MLPG3, MLPG4, MLPG5, and MLPG6, respectively. Six different MLPG methods derived from six different nodal-based local test functions are also selected. The test function over a local sub-domain is the same as the weight function in the moving least square approximation: The resultant is denoted as MLPG1. The test function over the local sub-domain is the collocation Dirac's delta function (collocation method): The resultant is denoted MLPG2. The test function over a local sub-domain is the same as the error function in the differential equation, using discrete least squares: The resultant is denoted MLPG3. The test function over local subdomain is the modified fundamental solution to the differential equation (local boundary integral equation method): The resultant is denoted MLPG4. The test function over local sub-domain is the Heaviside step function (constant over each local sub-domain): The resultant is denoted as MLPG5. The test function over local sub-domain is identical to the trial function (Galerkin method): The resultant is denoted as MLPG6. The MLPG2 does not involve any numerical integration to generate the global stiffness matrix, thus it is the simplest form of the meshless method. The MLPG4 does not involve the domain integral to generate the stiffness matrix but does involve a singular integral. The MLPG1, MLPG3 and MLPG6 methods involve domain integrals to generate the stiffness matrix, which is difficult in meshless methods due to the complexity of the integrand, especially for the MLPG3 and MLPG6. The MLPG5 does not involve any domain and singular integrals to generate the global stiffness matrix; it only involves a regular boundary integral [12].

The MLPG method has been applied to various problems in different fields. But also one found that constructing shape functions is one of the most important problems in the MLPG method because of the difficulty in implementing some essential boundary conditions [13]. There are many methods for constructing shape functions such as the moving least squares (MLS) method, the weighted least squares (WLS) method and the moving Kriging interpolation (MKI) method. In this paper, the MLPG method based on the moving Kriging is developed to solve the system of nonlinear partial differential equations. The moving Kriging for constructing shape function has the Kronecker delta property, which is a good property for constructing the shape function [14].

The aim of this paper is to study a numerical approximation of the HIV model in fractional derivatives of Caputo's definition which is used in the meshless local Petrov- 
Galerkin (MLPG) method in the field of numerical analysis. The outline of this paper is organized as follows: Sect. 1 is the review of the previous models proposed in previous work, Sect. 2 gives an idea about the generalized forward difference formula about the time fractional derivative equation in Caputo's sense. Then Sect. 3 presents the idea of generalized MLPG method for solving fractional order differential equations. In Sect. 4, a stability analysis of the infection-free steady state based on the eigenvalue of the matrix is presented. In Sect. 5, the numerical examination is given to support the proposed scheme formula. Finally, the results of this study can be concluded in Sect. 6 .

\section{Approximation of Caputo fractional order derivative}

The approximation solution of the time fractional derivative in Caputo's sense is shown in this section. The first-order approximation formula for computation of the time fractional derivative of order $\alpha$ is based on a quadrature rule and finite difference method $[15,16]$. The Caputo fractional derivative is presented as follows.

Definition 1 The fractional derivative of $f(t)$ in Caputo's sense is defined as

$$
D_{t}^{\alpha} f(t)=\frac{1}{\Gamma(n-\alpha)} \int_{0}^{t}(t-\tau)^{n-\alpha-1} \frac{d^{n} f(\tau)}{d \tau^{n}} d \tau ; \quad n-1<\alpha<n, n \in N, t>0,
$$

where $\Gamma(\cdot)$ denotes the gamma function. For $n=1$, a numerical approximation based upon the Caputo fractional derivative as Eq. (2.2):

$$
D_{t}^{\alpha} f(t)=\frac{1}{\Gamma(1-\alpha)} \int_{0}^{t}(t-\tau)^{-\alpha} \frac{d f(\tau)}{d \tau} d \tau ; \quad 0<\alpha<1, t>0,
$$

for some positive integer $N$, the grid size in time for finite difference technique is defined by $\Delta t=\frac{T}{N}$. The grid points in the time interval $[0, T]$ are labeled $t_{j}=j \Delta t ; j=0,1,2, \ldots, N$. The value of the function $f$ at the grid point is $f^{j}=f\left(t_{j}\right)$. We have

$$
D_{t}^{\alpha} f\left(t_{n}\right)=\frac{1}{\Gamma(1-\alpha)} \int_{t_{0}}^{t_{n}}\left(t_{n}-\tau\right)^{-\alpha} \frac{d f(\tau)}{d \tau} d \tau ; \quad 0<\alpha<1
$$

By $\int_{t_{0}}^{t_{n}} f(y) d y=\sum_{j=0}^{n-1} \int_{t_{j}}^{t_{j+1}} f(y) d y$, Eq. (2.3) can be rewritten as Eq. (2.4):

$$
D_{t}^{\alpha} f\left(t_{n}\right)=\frac{1}{\Gamma(1-\alpha)} \sum_{j=0}^{n-1} \int_{t_{j}}^{t_{j+1}}\left(t_{n}-\tau\right)^{-\alpha} \frac{d f(\tau)}{d \tau} d \tau .
$$

The approximation formula at the time level $n$ can be obtained as Eq. (2.5):

$$
\begin{aligned}
D_{t}^{\alpha} f\left(t_{n}\right) & =\frac{1}{\Gamma(1-\alpha)} \sum_{j=0}^{n-1} \int_{t_{j}}^{t_{j+1}}\left(t_{n}-\tau\right)^{-\alpha}\left(\frac{f^{j+1}-f^{j}}{\Delta t}+O(\Delta t)\right) d \tau \\
& =\frac{1}{\Gamma(1-\alpha)} \sum_{j=0}^{n-1} \int_{(j) \Delta t}^{(j+1) \Delta t}(n \Delta t-\tau)^{-\alpha}\left(\frac{f^{j+1}-f^{j}}{\Delta t}+O(\Delta t)\right) d \tau \\
& =\frac{1}{\Gamma(1-\alpha)} \sum_{j=0}^{n-1}\left(\frac{f^{j+1}-f^{j}}{\Delta t}+O(\Delta t)\right)\left(\frac{(n \Delta t-j \Delta t)^{1-\alpha}-(n \Delta t-(j+1) \Delta t)^{1-\alpha}}{1-\alpha}\right)
\end{aligned}
$$




$$
\begin{aligned}
& =\frac{\Delta t^{1-\alpha}}{\Gamma(1-\alpha)} \sum_{j=0}^{n-1}\left(\frac{f^{j+1}-f^{j}}{\Delta t}+O(\Delta t)\right)\left(\frac{(n-j)^{1-\alpha}-(n-j-1)^{1-\alpha}}{1-\alpha}\right) \\
& =\frac{\Delta t^{1-\alpha}}{\Gamma(2-\alpha)} \sum_{j=0}^{n-1}\left(\frac{f^{j+1}-f^{j}}{\Delta t}+O(\Delta t)\right) d_{j},
\end{aligned}
$$

where $\Gamma(z+1)=\mathrm{z} \Gamma(z)$ and $d_{j}=(n-j)^{1-\alpha}-(n-j-1)^{1-\alpha}$. Then defining the indices $n-j$ as $k$, which $d_{n-k}=k^{1-\alpha}-(k-1)^{1-\alpha}$. Thus Eq. (2.5) can be written as Eq. (2.6):

$$
\begin{aligned}
D_{t}^{\alpha} f\left(t_{n}\right) & =\frac{\Delta t^{1-\alpha}}{\Gamma(2-\alpha)} \sum_{k=1}^{n}\left(\frac{f^{n-k+1}-f^{n-k}}{\Delta t}+O(\Delta t)\right) d_{n-k} \\
& =\frac{\Delta t^{-\alpha}}{\Gamma(2-\alpha)} \sum_{k=1}^{n}\left(f^{n-k+1}-f^{n-k}\right) d_{n-k}+\frac{O\left(\Delta t^{2-\alpha}\right)}{\Gamma(2-\alpha)} \sum_{k=1}^{n} d_{n-k},
\end{aligned}
$$

Letting $D_{t}^{\alpha} f\left(t_{n}\right)=\mathfrak{D}_{t}^{\alpha} f\left(t_{n}\right)+O(\Delta t)$ and $\sigma_{\alpha}=\frac{\Delta t^{-\alpha}}{\Gamma(2-\alpha)}$. The first-order approximation formula of the time fractional derivative in Caputo's sense is given as Eq. (2.7):

$$
\mathfrak{D}_{t}^{\alpha} f\left(t_{n}\right)=\sigma_{\alpha} \sum_{k=1}^{n}\left(f^{n-k+1}-f^{n-k}\right) d_{n-k}
$$

\section{The spatial discretization}

This section demonstrates the approximation solution of the fractional HIV model for the spatial discretization. In the MLPG method, the moving Kriging interpolation are employed to construct the shape function which employs the Kronecker delta property [13, 14]. The Dirac delta function is applied in the local weak form as the test function [17]. Equations (1.1)-(1.3) can be transformed into fractional order differential equations of order $\alpha$ as follows:

$$
\begin{aligned}
& \frac{\partial^{\alpha} p(\mathbf{x}, t)}{\partial t^{\alpha}}-\lambda(\mathbf{x})+\beta_{1}(\mathbf{x}) p(\mathbf{x}, t) r(\mathbf{x}, t)+\beta_{2}(\mathbf{x}) p(\mathbf{x}, t) q(\mathbf{x}, t)+a(\mathbf{x}) p(\mathbf{x}, t)=0, \\
& \frac{\partial^{\alpha} q(\mathbf{x}, t)}{\partial t^{\alpha}}-\beta_{1}(\mathbf{x}) p(\mathbf{x}, t) r(\mathbf{x}, t)-\beta_{2}(\mathbf{x}) p(\mathbf{x}, t) q(\mathbf{x}, t)+b(\mathbf{x}) q(\mathbf{x}, t)=0, \\
& \frac{\partial^{\alpha} r(\mathbf{x}, t)}{\partial t^{\alpha}}-\mathcal{D} \Delta r(\mathbf{x}, t)-k(\mathbf{x}) q(\mathbf{x}, t)+m(\mathbf{x}) r(\mathbf{x}, t)=0,
\end{aligned}
$$

for $(\mathbf{x}, t) \in \Omega \times(0, \infty) ; \Omega \in \mathbb{R}^{d}, d=1,2,3$ with the homogeneous Neumann boundary condition $\frac{\partial r(\mathbf{x}, t)}{\partial \mathbf{n}}=0 ; \mathbf{x} \in \partial \Omega, t>0$ where $\mathbf{n}$ denotes an outward unit normal to $\partial \Omega$ and we have initial conditions $p(\mathbf{x}, 0)=p^{0}(\mathbf{x}) \geq 0, q(\mathbf{x}, 0)=q^{0}(\mathbf{x}) \geq 0, r(\mathbf{x}, 0)=r^{0}(\mathbf{x}) \geq 0 ; \mathbf{x} \in \Omega$.

Create the local weak form over local sub-domains, $\Omega_{S}$, which is a small region taken for each node in the global domain, $\Omega$. By multiplying the test function $w_{i}$ to Eqs. (3.1)-(3.3) and then integrating over a local sub-domains associated with the point $\mathbf{x}_{i} ; i=1,2,3, \ldots, N$ where $N$ is the number of nodes in the sub-domain, the result can be written as follows:

$$
\begin{aligned}
& \int_{\Omega_{S}^{i}}\left(\frac{\partial^{\alpha} p(\mathbf{x}, t)}{\partial t^{\alpha}}-\lambda(\mathbf{x})+\beta_{1}(\mathbf{x}) p(\mathbf{x}, t) r(\mathbf{x}, t)+\beta_{2}(\mathbf{x}) p(\mathbf{x}, t) q(\mathbf{x}, t)+a(\mathbf{x}) p(\mathbf{x}, t)\right) w_{i}(\mathbf{x}) d \Omega \\
& \quad=0
\end{aligned}
$$




$$
\begin{aligned}
& \int_{\Omega_{S}^{i}}\left(\frac{\partial^{\alpha} q(\mathbf{x}, t)}{\partial t^{\alpha}}-\beta_{1}(\mathbf{x}) p(\mathbf{x}, t) r(\mathbf{x}, t)-\beta_{2}(\mathbf{x}) p(\mathbf{x}, t) q(\mathbf{x}, t)+b(\mathbf{x}) q(\mathbf{x}, t)\right) w_{i}(\mathbf{x}) d \Omega \\
& \quad=0 \\
& \int_{\Omega_{S}^{i}}\left(\frac{\partial^{\alpha} r(\mathbf{x}, t)}{\partial t^{\alpha}}-\mathcal{D} \Delta r(\mathbf{x}, t)-k(\mathbf{x}) q(\mathbf{x}, t)+m(\mathbf{x}) r(\mathbf{x}, t)\right) w_{i}(\mathbf{x}) d \Omega=0,
\end{aligned}
$$

substituting $p(\mathbf{x}, t), q(\mathbf{x}, t)$ and $r(\mathbf{x}, t)$ in Eqs. (3.4)-(3.6) for the trial functions $p^{h}(\mathbf{x}, t)$, $q^{h}(\mathbf{x}, t)$ and $r^{h}(\mathbf{x}, t)$, respectively. Equations (3.4)-(3.6) can be presented as follows:

$$
\begin{aligned}
& \sum_{j=1}^{N}\left(\int_{\Omega_{S}^{i}} \phi_{j}(\mathbf{x}) w_{i}(\mathbf{x}) d \Omega\right) \mathfrak{D}_{t}^{\alpha} \hat{p}_{j}(t)-\int_{\Omega_{S}^{i}} \lambda(\mathbf{x}) w_{i}(\mathbf{x}) d \Omega \\
& +\sum_{j=1}^{N}\left(\int_{\Omega_{S}^{i}} \beta_{1}(\mathbf{x}) r(\mathbf{x}, t) \phi_{j}(\mathbf{x}) w_{i}(\mathbf{x}) d \Omega\right) \hat{p}_{j}(t) \\
& +\sum_{j=1}^{N}\left(\int_{\Omega_{S}^{i}} \beta_{2}(\mathbf{x}) q(\mathbf{x}, t) \phi_{j}(\mathbf{x}) w_{i}(\mathbf{x}) d \Omega\right) \hat{p}_{j}(t) \\
& +\sum_{j=1}^{N}\left(\int_{\Omega_{S}^{i}} a(\mathbf{x}) \phi_{j}(\mathbf{x}) w_{i}(\mathbf{x}) d \Omega\right) \hat{p}_{j}(t)=0 \\
& \sum_{j=1}^{N}\left(\int_{\Omega_{S}^{i}} \phi_{j}(\mathbf{x}) w_{i}(\mathbf{x}) d \Omega\right) \mathfrak{D}_{t}^{\alpha} \hat{q}_{j}(t)-\int_{\Omega_{S}^{i}} \beta_{1}(\mathbf{x}) r(\mathbf{x}, t) \phi_{j}(\mathbf{x}) w_{i}(\mathbf{x}) d \Omega \\
& -\sum_{j=1}^{N}\left(\int_{\Omega_{S}^{i}} \beta_{2}(\mathbf{x}) p(\mathbf{x}, t) \phi_{j}(\mathbf{x}) w_{i}(\mathbf{x}) d \Omega\right) \hat{q}_{j}(t) \\
& +\sum_{j=1}^{N}\left(\int_{\Omega_{S}^{i}} b(\mathbf{x}) \phi_{j}(\mathbf{x}) w_{i}(\mathbf{x}) d \Omega\right) \hat{q}_{j}(t)=0 \\
& \sum_{j=1}^{N}\left(\int_{\Omega_{S}^{i}} \phi_{j}(\mathbf{x}) w_{i}(\mathbf{x}) d \Omega\right) \mathfrak{D}_{t}^{\alpha} \hat{r}_{j}(t)-\sum_{j=1}^{N}\left(\int_{\Omega_{S}^{i}} \mathcal{D} \phi_{j, \mathbf{x x}}(\mathbf{x}) w_{i}(\mathbf{x}) d \Omega\right) \hat{r}_{j}(t) \\
& -\int_{\Omega_{S}^{i}} k(\mathbf{x}) \phi_{j}(\mathbf{x}) w_{i}(\mathbf{x}) d \Omega+\sum_{j=1}^{N}\left(\int_{\Omega_{S}^{i}} m(\mathbf{x}) \phi_{j}(\mathbf{x}) w_{i}(\mathbf{x}) d \Omega\right) \hat{r}_{j}(t)=0,
\end{aligned}
$$

where $p^{h}(\mathbf{x}, t)=\sum_{j=1}^{N} \phi_{j}(\mathbf{x}) \hat{p}_{j}(t), q^{h}(\mathbf{x}, t)=\sum_{j=1}^{N} \phi_{j}(\mathbf{x}) \hat{q}_{j}(t)$ and $r^{h}(\mathbf{x}, t)=\sum_{j=1}^{N} \phi_{j}(\mathbf{x}) \hat{r}_{j}(t)$. The shape function, $\phi_{j}$, is constructed by a moving Kriging interpolation that satisfies the Kronecker delta property. Using MLPG2, the test function in the local weak form is chosen as Dirac delta function. The test function is defined with significance for each node in subdomain as a unit value while all other nodes are set as zero. Integrating Eqs. (3.7)-(3.9) over sub-domain $\Omega_{S}^{i}$, the results can be shown as follows:

$$
\begin{aligned}
& \sum_{j=1}^{N} \phi_{j}\left(\mathbf{x}_{i}\right) \mathfrak{D}_{t}^{\alpha} \hat{p}_{j}(t)+\sum_{j=1}^{N} \beta_{1}\left(\mathbf{x}_{i}\right) r\left(\mathbf{x}_{i}, t\right) \phi_{j}\left(\mathbf{x}_{i}\right) \hat{p}_{j}(t)+\sum_{j=1}^{N} \beta_{2}\left(\mathbf{x}_{i}\right) q\left(\mathbf{x}_{i}, t\right) \phi_{j}\left(\mathbf{x}_{i}\right) \hat{p}_{j}(t) \\
& \quad+\sum_{j=1}^{N} a\left(\mathbf{x}_{i}\right) \phi_{j}\left(\mathbf{x}_{i}\right) \hat{p}_{j}(t)=\lambda\left(\mathbf{x}_{i}\right),
\end{aligned}
$$




$$
\begin{aligned}
& \sum_{j=1}^{N} \phi_{j}\left(\mathbf{x}_{i}\right) \mathfrak{D}_{t}^{\alpha} \hat{q}_{j}(t)-\sum_{j=1}^{N} \beta_{2}\left(\mathbf{x}_{i}\right) p\left(\mathbf{x}_{i}, t\right) \phi_{j}\left(\mathbf{x}_{i}\right) \hat{q}_{j}(t)+\sum_{j=1}^{N} b\left(\mathbf{x}_{i}\right) \phi_{j}\left(\mathbf{x}_{i}\right) \hat{q}_{j}(t) \\
& \quad=\beta_{1}\left(\mathbf{x}_{i}\right) p\left(\mathbf{x}_{i}, t\right) r\left(\mathbf{x}_{i}, t\right), \\
& \sum_{j=1}^{N} \phi_{j}\left(\mathbf{x}_{i}\right) \mathfrak{D}_{t}^{\alpha} \hat{r}_{j}(t)-\sum_{j=1}^{N} \mathcal{D} \phi_{j, \mathbf{x}}\left(\mathbf{x}_{i}\right) \hat{r}_{j}(t)+\sum_{j=1}^{N} m\left(\mathbf{x}_{i}\right) \phi_{j}\left(\mathbf{x}_{i}\right) \hat{r}_{j}(t)=k\left(\mathbf{x}_{i}\right) q\left(\mathbf{x}_{i}, t\right) .
\end{aligned}
$$

Equations (3.10)-(3.12) can be written in a matrix form as Eq. (3.13):

$$
A \mathfrak{D}_{t}^{\alpha} U+B(U) U=C(U)
$$

where

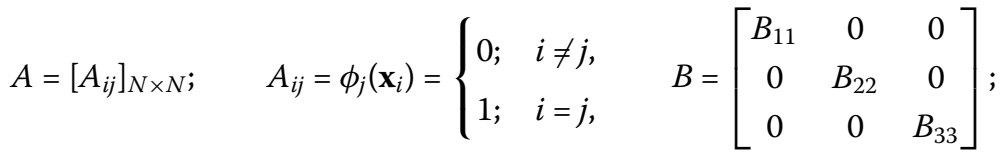

$$
\begin{aligned}
& B_{11}=\left[\beta_{1}\left(\mathbf{x}_{i}\right) r\left(\mathbf{x}_{i}, t\right) \phi_{j}\left(\mathbf{x}_{i}\right)+\beta_{2}\left(\mathbf{x}_{i}\right) q\left(\mathbf{x}_{i}, t\right) \phi_{j}\left(\mathbf{x}_{i}\right)+a\left(\mathbf{x}_{i}\right) \phi_{j, \mathbf{x}}\left(\mathbf{x}_{i}\right)\right], \\
& B_{22}=\left[b\left(\mathbf{x}_{i}\right) \phi_{j}\left(\mathbf{x}_{i}\right)-\beta_{2}\left(\mathbf{x}_{i}\right) p\left(\mathbf{x}_{i}, t\right) \phi_{j}\left(\mathbf{x}_{i}\right)\right], \quad B_{33}=\left[m\left(\mathbf{x}_{i}\right) \phi_{j}\left(\mathbf{x}_{i}\right)-\mathcal{D} \phi_{j, \mathbf{x x}}\left(\mathbf{x}_{i}\right)\right], \\
& C=\left[\begin{array}{lll}
C_{1} & C_{2} & C_{3}
\end{array}\right]^{T} ; \quad C_{1}=\left[\lambda\left(\mathbf{x}_{i}\right)\right], \\
& C_{2}=\left[\beta_{1}\left(\mathbf{x}_{i}\right) r\left(\mathbf{x}_{i}, t\right) \phi_{j}\left(\mathbf{x}_{i}\right)\right], \quad C_{3}=\left[k\left(\mathbf{x}_{i}\right) \phi_{j}\left(\mathbf{x}_{i}\right)\right], \\
& U=\left[\begin{array}{lll}
\hat{P} & \hat{Q} & \hat{R}
\end{array}\right]^{T} ; \quad \hat{P}=\left[\begin{array}{llll}
\hat{p}_{1} & \hat{p}_{2} & \cdots & \hat{p}_{N}
\end{array}\right]^{T}, \\
& \hat{Q}=\left[\begin{array}{llll}
\hat{q}_{1} & \hat{q}_{2} & \cdots & \hat{q}_{N}
\end{array}\right]^{T,} \quad \hat{R}=\left[\begin{array}{llll}
\hat{r}_{1} & \hat{r}_{2} & \cdots & \hat{r}_{N}
\end{array}\right]^{T} \text {. }
\end{aligned}
$$

Since the shape function constructed by the moving Kriging interpolation satisfies the Kronecker delta property, $A$ is an identity matrix. Next, Eq. (3.13) is approximated by discretizing with the time level $n$ of Eq. (3.13). By substituting the approximation formula of time fractional derivative in Caputo's sense, $\mathfrak{D}_{t}^{\alpha} U$. Equation (3.14) is an approximate solution of computing the vector $U$ at the time level $n$ as Eq. (3.14):

$$
\sigma_{\alpha} \sum_{k=1}^{n}\left(U^{n-k+1}-U^{n-k}\right) d_{n-k}+B\left(U^{n}\right) U^{n}=C\left(U^{n}\right)
$$

Let $B^{n}=B\left(U^{n}\right)$ and $C^{n}=C\left(U^{n}\right)$. For $n=1$, at the first-time level of Eq. (3.14) can be presented as follows:

$$
\begin{aligned}
& \sigma_{\alpha}\left(U^{1}-U^{0}\right) d_{0}+B^{1} U^{1}=C^{1}, \\
& U^{1}-U^{0}+\frac{1}{\sigma_{\alpha} d_{0}} B^{1} U^{1}=\frac{1}{\sigma_{\alpha} d_{0}} C^{1}, \\
& U^{1}+\frac{1}{\sigma_{\alpha} d_{0}} B^{1} U^{1}=U^{0}+\frac{1}{\sigma_{\alpha} d_{0}} C^{1}, \\
& \left(I+\frac{1}{\sigma_{\alpha} d_{0}} B^{1}\right) U^{1}=U^{0}+\frac{1}{\sigma_{\alpha} d_{0}} C^{1}, \\
& U^{1}=\left(I+\frac{1}{\sigma_{\alpha} d_{0}} B^{1}\right)^{-1}\left(U^{0}+\frac{1}{\sigma_{\alpha} d_{0}} C^{1}\right) .
\end{aligned}
$$


For $n \geq 2$, the time level $n$ corresponding to Eq. (3.19) can be written as follows:

$$
\begin{aligned}
& \sigma_{\alpha}\left(U^{n}-U^{n-1}\right) d_{n-1}+\sigma_{\alpha} \sum_{k=2}^{n}\left(U^{n-k+1}-U^{n-k}\right) d_{n-k}+B^{n} U^{n}=C^{n}, \\
& U^{n}-U^{n-1}+\frac{1}{d_{n-1}} \sum_{k=2}^{n}\left(U^{n-k+1}-U^{n-k}\right) d_{n-k}+\frac{1}{\sigma_{\alpha} d_{n-1}} B^{n} U^{n}=\frac{1}{\sigma_{\alpha} d_{n-1}} C^{n}, \\
& U^{n}+\frac{1}{\sigma_{\alpha} d_{n-1}} B^{n} U^{n}=U^{n-1}-\frac{1}{d_{n-1}} \sum_{k=2}^{n}\left(U^{n-k+1}-U^{n-k}\right) d_{n-k}+\frac{1}{\sigma_{\alpha} d_{n-1}} C^{n}, \\
& \left(I+\frac{1}{\sigma_{\alpha} d_{n-1}} B^{n}\right) U^{n}=U^{n-1}-\frac{1}{d_{n-1}} \sum_{k=2}^{n}\left(U^{n-k+1}-U^{n-k}\right) d_{n-k}+\frac{1}{\sigma_{\alpha} d_{n-1}} C^{n}, \\
& U^{n}=\left(I+\frac{1}{\sigma_{\alpha} d_{n-1}} B^{n}\right)^{-1}\left(U^{n-1}-\frac{1}{d_{n-1}} \sum_{k=2}^{n}\left(U^{n-k+1}-U^{n-k}\right) d_{n-k}+\frac{1}{\sigma_{\alpha} d_{n-1}} C^{n}\right),
\end{aligned}
$$

where $G^{n}=\left(I+\frac{1}{\sigma_{\alpha} d_{n-1}} B^{n}\right)^{-1}$. The matrix $G^{n}$ must be non-singular.

In order to find the approximated solution at the time level $n$, we need to know the approximate solution at every time level. The previous time step is used to approximate the solution of the next time step with appropriate small time increment. Alternatively, the use of a conventional time as fixed point iteration can improve the accuracy of the solution when dealing with nonlinearities [18]. We can rewrite nonlinear Eq. (3.24) in the form of Eq. (3.25):

$$
\begin{aligned}
U^{n, \gamma+1}= & \left(I+\frac{1}{\sigma_{\alpha} d_{n-1}} B\left(U^{n, \gamma}\right)\right)^{-1} \\
& \times\left(U^{n-1}-\frac{1}{d_{n-1}} \sum_{k=2}^{n}\left(U^{n-k+1}-U^{n-k}\right) d_{n-k}+\frac{1}{\sigma_{\alpha} d_{n-1}} C^{n}\right),
\end{aligned}
$$

where $n$ denotes the time level and $\gamma$ denotes the number of iterations for solving nonlinear system. In the first-time level, $\gamma=0$, we pick $U^{n}$ as initial guess $U^{n, 0}=U^{n-1}$. When the solution is updated, the numerical results from the previous time is used as the default for the next replication. The process is repeated indefinitely until the condition of $\left\|U^{n, \gamma+1}-U^{n, \gamma}\right\|_{\infty} \leq \varepsilon$ is satisfied. In this paper, $\varepsilon$ is selected as $0.5 \times 10^{-8}$. That is, the process is repeated until the computed values for all points satisfy the stopping criteria.

\section{Stability analysis of the numerical scheme}

In this section, a stability analysis based on the eigenvalue of the matrix is applied to verify the condition of stability of the MLPG method [18]. A small perturbation at each $n$th time level is set as Eq. (4.1):

$$
e^{n}=U^{n}-\hat{U}^{n}
$$

where $U^{n}$ denotes the exact solution at the time level $n$ and $\hat{U}^{n}$ represents the approximate solution at the time level $n$. So that from Eq. (3.24) can be rewritten as Eq. (4.2):

$$
e^{n}=G^{n}\left(e^{n-1}-\frac{1}{d_{n-1}} \sum_{k=2}^{n}\left(e^{n-k+1}-e^{n-k}\right) d_{n-k}\right) .
$$


It can be seen that stability can be assumed, if all eigenvalues of the matrix $G^{n}=$ $\left(I+\frac{1}{\sigma_{\alpha} d_{n-1}} B^{n}\right)^{-1}$ satisfy the condition $\left|\frac{\sigma_{\alpha} d_{n-1}}{\lambda}\right| \leq 1$ where $\lambda$ is the eigenvalue of the matrices $B$. Equation (4.1) will be stable if $e^{n}$ is bounded as $n$ increases indefinitely. That is, there exists a positive number $M$ such that $\left\|G^{n}\right\| \leq M$. Then $\left\|e^{n}\right\| \leq M\left\|e^{0}\right\|$. Consider Eq. (4.2) in the case of $n=1$ and $n \geq 2$. We prove that $\left\|e^{n}\right\| \leq\left\|G^{n}\right\|\left\|e^{0}\right\| ; \forall n \in N$. When $n=1$, it is easy to see that $e^{1}=G^{1} e^{0}$. Taking the norm of both sides and making use the compatibility condition for matrix norms and the triangle inequality, it can be written as $\left\|e^{1}\right\| \leq\left\|G^{1}\right\|\left\|e^{0}\right\|$.

If $\left\|G^{1}\right\| \leq 1$, then $\left\|e^{1}\right\| \leq\left\|e^{0}\right\|$. In the case of $n \geq 2$, it can be done by means of mathematical induction. Equation (4.2) be rearranged as Eq. (4.3):

$$
\begin{aligned}
e^{n} & =G^{n}\left(e^{n-1}-\frac{1}{d_{n-1}} \sum_{k=2}^{n}\left(e^{n-k+1}-e^{n-k}\right) d_{n-k}\right) \\
& =G^{n}\left(e^{n-1}-\frac{1}{d_{n-1}} \sum_{k=2}^{n} e^{n-k+1} d_{n-k}+\frac{1}{d_{n-1}} \sum_{k=2}^{n} e^{n-k} d_{n-k}\right) \\
& =G^{n}\left(e^{n-1}-\frac{1}{d_{n-1}} e^{n-1} d_{n-2}-\frac{1}{d_{n-1}} \sum_{k=3}^{n} e^{n-k+1} d_{n-k}+\frac{1}{d_{n-1}} e^{0} d_{0}+\frac{1}{d_{n-1}} \sum_{k=2}^{n-1} e^{n-k} d_{n-k}\right) \\
& =G^{n}\left(e^{n-1}-\frac{d_{n-2}}{d_{n-1}} e^{n-1}-\frac{1}{d_{n-1}} \sum_{k=2}^{n-1} e^{n-k} d_{n-k-1}+\frac{d_{0}}{d_{n-1}} e^{0}+\frac{1}{d_{n-1}} \sum_{k=2}^{n-1} e^{n-k} d_{n-k}\right) \\
& =G^{n}\left(e^{n-1}-\frac{d_{n-2}}{d_{n-1}} e^{n-1}+\frac{1}{d_{n-1}} \sum_{k=2}^{n-1} e^{n-k}\left(d_{n-k}-d_{n-k-1}\right)+\frac{d_{0}}{d_{n-1}} e^{0}\right) \\
& =G^{n}\left(\left(1-\frac{d_{n-2}}{d_{n-1}}\right) e^{n-1}+\frac{d_{n-2}}{d_{n-1}} e^{n-2}\right) .
\end{aligned}
$$

Taking the norm of both sides of Eq. (4.3) and making use of the compatibility condition for matrix norms and the triangle inequality, it can be written as Eq. (4.4):

$$
\left\|e^{n}\right\| \leq\left\|G^{n}\right\|\left(\left|1-\frac{d_{n-2}}{d_{n-1}}\right|\left\|e^{n-1}\right\|+\left|\frac{d_{n-2}}{d_{n-1}}\right|\left\|e^{n-2}\right\|\right) .
$$

Since $d_{n-k}=k^{1-\alpha}-(k-1)^{1-\alpha} ; k=1,2,3, \ldots, n$, and $\left\{d_{j}\right\}$ is the increasing sequence, we have $\frac{d_{i}}{d_{j}}<1$ for $i<j$. When $n=2$, it can be defined as Eq. (4.5):

$$
\begin{aligned}
\left\|e^{2}\right\| & \leq\left\|G^{2}\right\|\left(\left|1-\frac{d_{0}}{d_{1}}\right|\left\|e^{1}\right\|+\left|\frac{d_{0}}{d_{1}}\right|\left\|e^{0}\right\|\right) \\
& \leq\left\|G^{2}\right\|\left(\left|1-\frac{d_{0}}{d_{1}}\right|\left\|G^{1}\right\|\left\|e^{0}\right\|+\left|\frac{d_{0}}{d_{1}}\right|\left\|e^{0}\right\|\right) \\
& \leq\left\|G^{2}\right\|\left\|e^{0}\right\|\left(\left|1-\frac{d_{0}}{d_{1}}\right|\left\|G^{1}\right\|+\left|\frac{d_{0}}{d_{1}}\right|\right) \\
& \leq\left\|G^{2}\right\|\left\|e^{0}\right\|\left(\left|1-\frac{d_{0}}{d_{1}}\right|+\left|\frac{d_{0}}{d_{1}}\right|\right) \\
& \leq\left\|G^{2}\right\|\left\|e^{0}\right\| .
\end{aligned}
$$


If $\left\|G^{m}\right\| \leq 1$, then $\left\|e^{m}\right\| \leq\left\|e^{0}\right\|$. Suppose that $\left\|e^{m}\right\| \leq\left\|G^{m}\right\|\left\|e^{0}\right\| ; \forall m \geq 2$; we have to show that $\left\|e^{m+1}\right\| \leq\left\|G^{m+1}\right\|\left\|e^{0}\right\|$ which can be written as Eq. (4.6):

$$
\begin{aligned}
\left\|e^{m+1}\right\| & \leq\left\|G^{m+1}\right\|\left(\left|1-\frac{d_{m-1}}{d_{m}}\right|\left\|e^{m}\right\|+\left|\frac{d_{m-1}}{d_{m}}\right|\left\|e^{m-1}\right\|\right) \\
& \leq\left\|G^{m+1}\right\|\left(\left|1-\frac{d_{m-1}}{d_{m}}\right|\left\|G^{m}\right\|\left\|e^{0}\right\|+\left|\frac{d_{m-1}}{d_{m}}\right|\left\|G^{m-1}\right\|\left\|e^{0}\right\|\right) \\
& \leq\left\|G^{m+1}\right\|\left\|e^{0}\right\|\left(\left|1-\frac{d_{m-1}}{d_{m}}\right|\left\|G^{m}\right\|+\left|\frac{d_{m-1}}{d_{m}}\right|\left\|G^{m-1}\right\|\right) \\
& \leq\left\|G^{m+1}\right\|\left\|e^{0}\right\|\left(\left|1-\frac{d_{m-1}}{d_{m}}\right|+\left|\frac{d_{m-1}}{d_{m}}\right|\right) \\
& \leq\left\|G^{m+1}\right\|\left\|e^{0}\right\| .
\end{aligned}
$$

If $\left\|G^{m+1}\right\|<1$, then $\left\|e^{m+1}\right\| \leq\left\|e^{0}\right\|$. Thus the proof is complete. The proof shows that the error made at each time level of calculation will be no more than the error made at the initial step as long as $\left\|G^{m}\right\| \leq 1$, which means all eigenvalues of the matrix $G^{n}=(I+$ $\left.\frac{1}{\sigma_{\alpha} d_{n-1}} B^{n}\right)^{-1}$ satisfy the condition $\left|\frac{\sigma_{\alpha} d_{n-1}}{\lambda}\right| \leq 1$ where $\lambda$ is the eigenvalue of the matrices $B$.

\section{Numerical experiments}

This section consists of two parts. The first part is to compare the numerical results of the integer order differetial equation and those of the fractional order differential equation. In the second part, the numerical simulations of the fractional order differential equation at different values of order alpha are performed.

\subsection{Comparison between the approximation solutions of integer order with fractional order differential equation}

In this part we attempt to address examples for two cases: $\beta_{1}$ is a constant and $\beta_{1}$ is a function that depends on the $\mathbf{x}$ value [1]. The approximation of the integer order differential equation will be compared with the approximation of the fractional order differential equation. Root mean square errors are used to make the comparison. We wish to confirm that the formula for the approximation of the proposed scheme corresponds to the approximation of the integer order differential equation.

Example 1 Consider the parameters of Eq. (3.24) to have the values $\alpha=0.99, \lambda=0.332$, $a=1, b=1, \beta_{2}=2, k=2, m=1, D=0.01$ and $\beta_{1}=0.47$. The initial conditions in this case are $p(\mathbf{x}, 0)=0.99, q(\mathbf{x}, 0)=0, r(\mathbf{x}, 0)=e^{-(\mathbf{x}-5)^{2}} \times 10^{-3}, \mathbf{x} \in \Omega=[0,10]$. From Fig. 1(a), it was found that the density of free virus $r(\mathbf{x}, t)$ became zero. Therefore this is a case free of infection. The approximation solution of the integer order differential equation by finite difference methods is shown in Fig. 1(b). The absolute error between the approximations solutions of the fractional order with the integer order differential equation is shown in Fig. 1(c). The values of the root mean square error between the approximation solutions of the fractional order with the integer order differential equation is shown in Fig. 1(d). It was found that the numerical results for the two schemes agree as well.

On the other hand, if $\beta_{1}=0.55$, then the density of free virus $r(\mathbf{x}, t)$ was unstable. The existence of the infection state is shown in Fig. 2. 

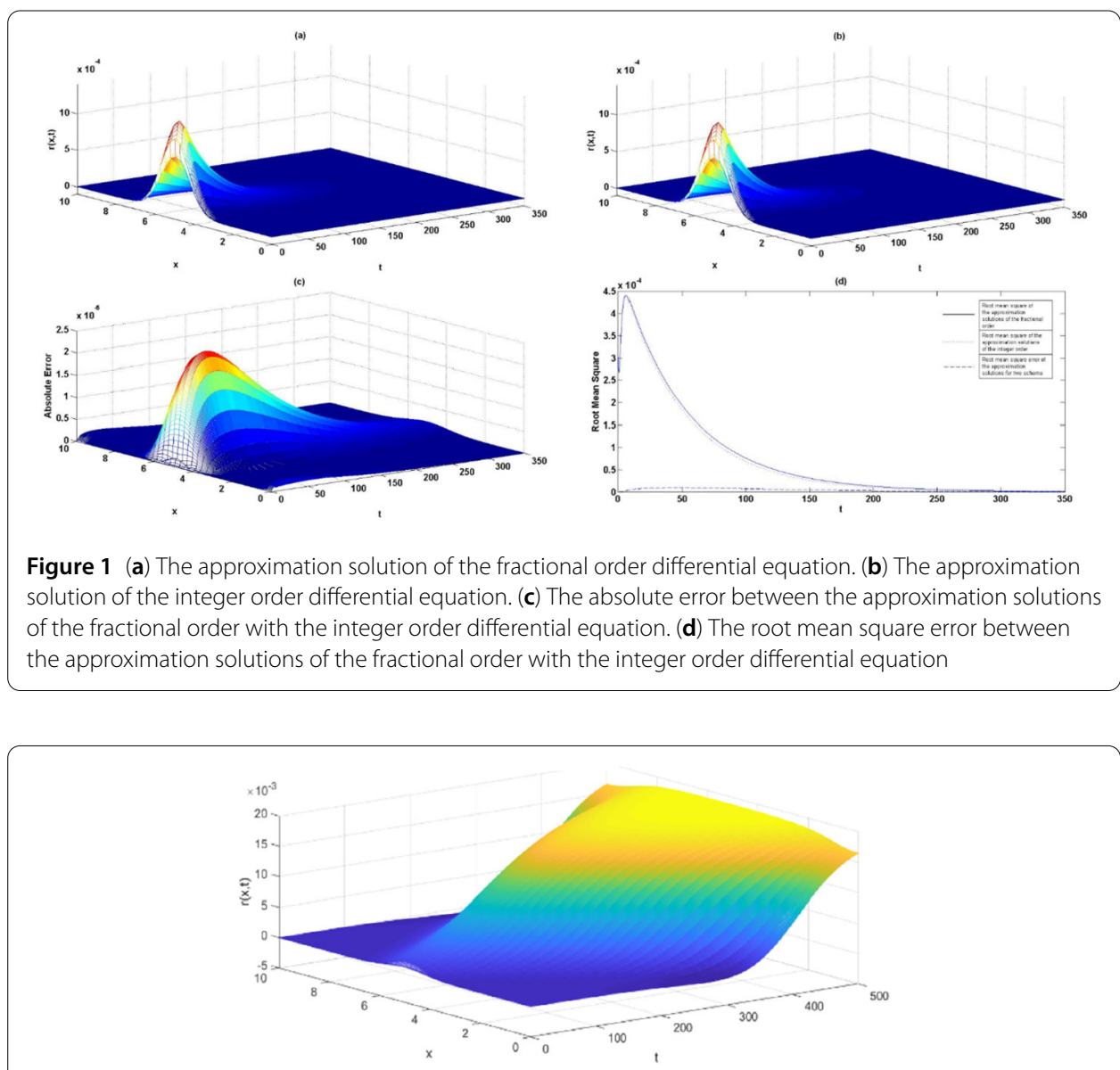

Figure 2 The approximation solution of the fractional order differential equation

Example 2 Let the parameters be $\alpha=0.99, \lambda=0.332, a=1, b=1, \beta_{2}=2, k=2, m=1$, $D=1 \times 10^{-5}$ and $\beta_{1}=\hat{\beta}\left(1+0.1 \sin \frac{9 \pi \mathbf{x}}{10}\right)$. The initial conditions in this case are $p(\mathbf{x}, 0)=$ 0.99, $q(\mathbf{x}, 0)=0, r(\mathbf{x}, 0)=e^{-(\mathbf{x}-5)^{2}} \times 10^{-3}, \mathbf{x} \in \Omega=[0,10]$. For $\hat{\beta}=0.45$, it is found that the density of free virus $r(\mathbf{x}, t)$ became zero. Therefore we have a case free of infection, shown in Fig. 3(a). The approximation solution of the integer order differential equation by finite difference methods shown in Fig. 3(b). The absolute error between the approximations solutions of the fractional order with the integer order differential equation is shown in Fig. 3(c). The values of the root mean square error between the approximation solutions of the fractional order with the integer order differential equation is shown in Fig. 3(d). It was found that the numerical results for the two schemes agree as well.

On the other hand, if $\hat{\beta}=0.50$, then the density of free virus $r(\mathbf{x}, t)$ is unstable. The existence of the infection state is shown in Fig. 4.

\subsection{Numerical simulation for different alpha values}

This section presents the numerical results for different alpha values. In Fig. 5(a)-(f) we illustrate the numerical solutions for the different orders of fractional differentiation at $\alpha=0.01,0.20,0.40,0.60,0.80,0.99$. In this case, $\lambda=0.332, a=1, b=1, \beta_{2}=2, k=2, m=1$, $D=0.01, \beta_{1}=0.47$. When setting the alpha values differently, it can be seen that the numerical results of the fractional order differential equations clearly explain the behavior of 

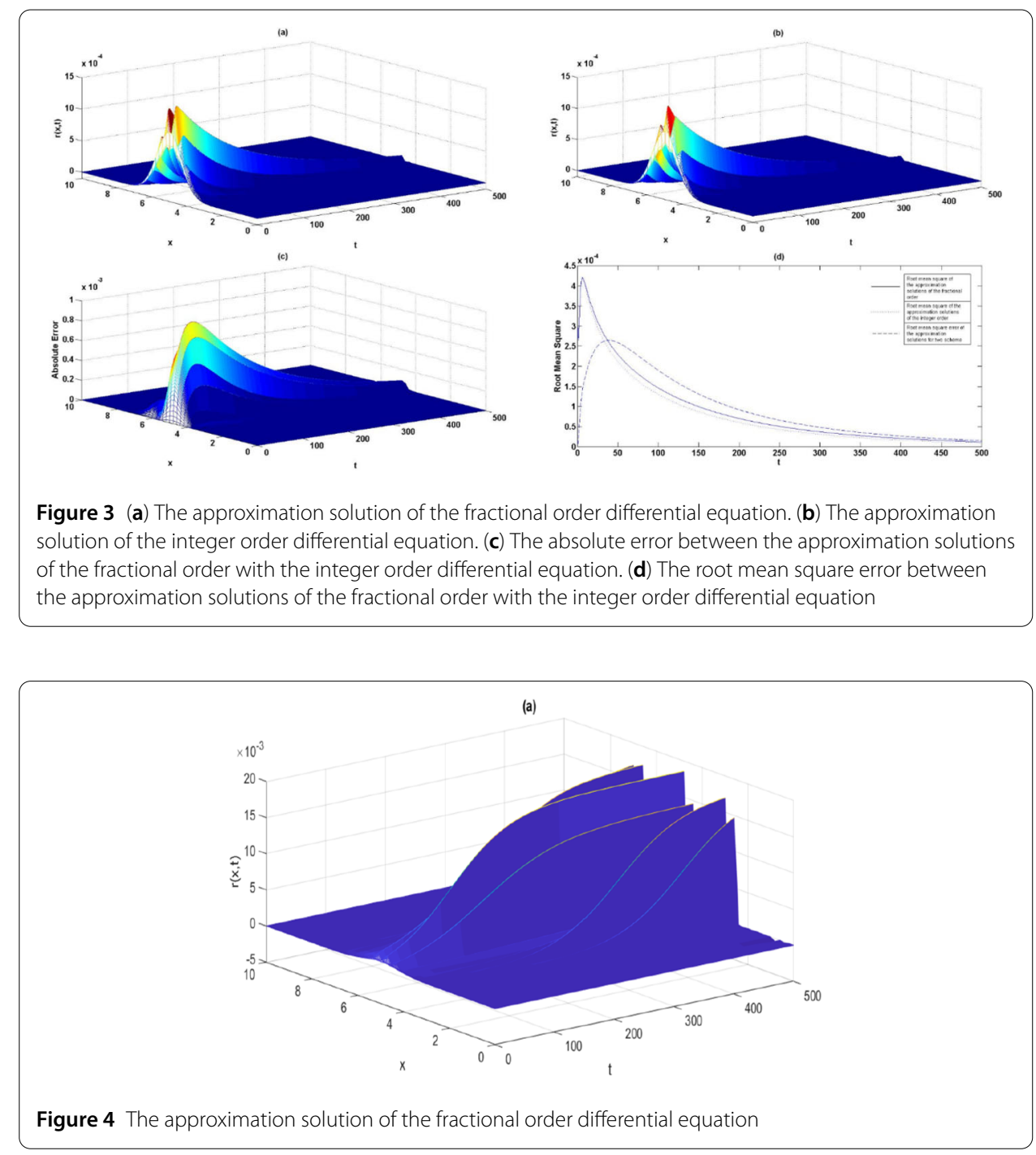

the free virus density $r(\mathbf{x}, t)$. For a short time period, lower fractional order solutions diffuse more slowly; however, as time increases the diffusion phenomena become apparent. This implies that the behavior of the virus density is more noticeable if the alpha value is decreased.

\section{Conclusion}

This paper is to meant explain the dynamics of the HIV model extended by the Caputo fractional derivative. The solution of a fractional HIV model with initial conditions is approximated by a meshless formulation since the trial and test functions can be chosen from different functional spaces. In this MLPG method, the moving Kriging interpolation is used to create the shape function which satisfies the Kronecker delta property. The test function in a local weak form is chosen such that the property of the Dirac delta function is satisfied. The MLPG method is truly a meshless method. Thus, it involves not only a meshless interpolation of trial functions, but it also includes a meshless integration of local weak forms. It does not require a mesh or background element. The MLPG method has an identity in selecting the trial and test functions including the size and shape of sub- 


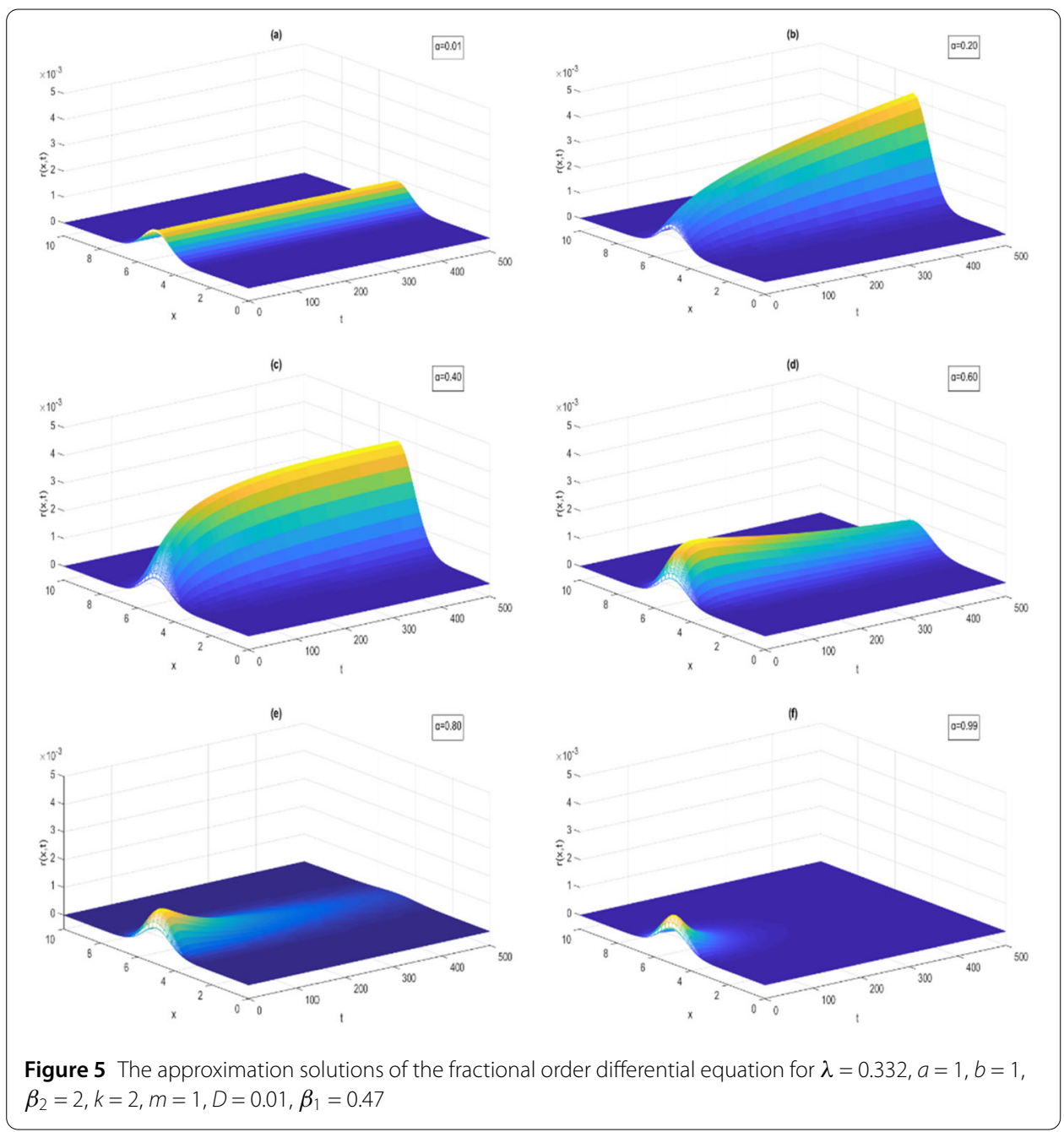

domains in the area. The MLPG method is more flexible and can handle problems easily compared with traditional mesh methods. Moreover, formulas with fixed-point iteration methods can improve the accuracy of the numerical result when a valid choice of $\Delta t$ can be selected. Comparing with the numerical result of finite difference methods, it can be found that the MLPG method for fractional order differential equations is equally reliable as the solution of the approximation of integer order differential equations. The effect of changing the value of alpha on the convergent rate of a free virus can be seen clearly when the value of alpha is small. It shows that fractional order differential equations represent the behavior of events more clearly than that of the integer order differential equations. The stability analysis shows that the reliability of the solution can be guaranteed by this theatrical study as shown in Sect. 4. The stability analysis using this technique is conducted based on the eigenvalues of the matrix. In addition, the problem is solved by various formulas based on temporal discretization with implicit finite difference. Using the iterative method, the stability and precision can be satisfied. Therefore, the results of this study provide an alternative useful approach. 
Funding

This research is supported by King Mongkut's University of Technology Thonburi (KMUTT) and Kanchanaburi Rajabhat University.

\section{Competing interests}

The authors declare that they have no competing interests.

\section{Authors' contributions}

The idea of this research was introduced by AL and KP. All authors contributed to the main results and numerical simulations. KP contributed to revising the manuscript. All authors read and approved the final manuscript.

\section{Publisher's Note}

Springer Nature remains neutral with regard to jurisdictional claims in published maps and institutional affiliations.

Received: 28 February 2019 Accepted: 22 August 2019 Published online: 03 September 2019

\section{References}

1. Wang, J., Yang, J., Kuniya, T.: Dynamics of a PDE viral infection model incorporating. J. Math. Anal. Appl. 444, 1452-1564 (2016)

2. Arafa, A., Rida, S., Khalil, M.: Fractional modeling dynamics of HIV and CD4 ${ }^{+}$T-cells during primary infection. Nonlinear Biomed. Phys. 6, 1 (2012)

3. Atluri, S.N., Zhu, T.: A new meshless local Petrov-Galerkin (MLPG) approach to nonlinear problems in computational modeling and simulation. Comput. Model. Simul. Eng. 3, 187-196 (1998)

4. Atluri, S.N., Zhu, T: A new meshless local Petrov-Galerkin (MLPG) approach in computational mechanics. Comput. Mech. 22, 117-127 (1998)

5. Atluri, S.N., Kim, H.G., Cho, J.Y.: A critical assessment of the truly meshless local Petrov-Galerkin (MLPG) and local boundary integral equation (LBIE) methods. Comput. Mech. 24, 348-372 (1999)

6. Lin, H., Atluri, S.N.: Meshless local Petrov-Galerkin (MPLG) method for convection-diffusion problems. CMES: Comput. Model. Eng. Sci. 1(2), 45-60 (2000)

7. Kim, H.G., Atluri, S.N.: Arbitrary placement of secondary nodes, and error control, in the meshless local Petrov-Galerkin (MPLG) method. CMES: Comput. Model. Eng. Sci. 1(3), 11-32 (2000)

8. Ching, H.K., Batra, R.C.: Determination of crack tip fields in linear elastostatics by the meshless local Petrov-Galerkin (MLPG) method. CMES: Comput. Model. Eng. Sci. 2(2), 273-290 (2001)

9. Lin, H., Atluri, S.N.: The meshless local Petrov-Galerkin (MPLG) method for solving incompressible Navier-Stokes equations. CMES: Comput. Model. Eng. Sci. 2(2), 117-142 (2001)

10. Gu, Y.T., Liu, G.R.: A meshless local Petrov-Galerkin (MLPG) formulation for static and free vibration analysis of thin plates. CMES: Comput. Model. Eng. Sci. 2(4), 463-476 (2001)

11. Long, S., Atluri, S.N.: A meshless local PetrovGalerkin (MLPG) method for solving the bending problem of a thin plate. CMES: Comput. Model. Eng. Sci. 3(1) (2002)

12. Atluri, N., Shen, S.: The meshless local Petrov-Galerkin method: a simple \& less-costly alternative to the finite element and boundary element methods. Comput. Model. Eng. Sci. 3(1), 11-51 (2002)

13. Phaochoo, P., Luadsong, A., Aschariyaphotha, N.: A numerical study of the European option by the MLPG method with moving kriging interpolation. SpringerPlus 5, 305 (2016)

14. Yimnak, K., Luadsong, A.: A local integral equation formulation based on moving kriging interpolation for solving coupled nonlinear reaction-diffusion equations. Adv. Math. Phys. 2014, Article ID 196041 (2014)

15. Atangana, A., Alqahtani, R.T.: Numerical approximation of the space-time Caputo-Fabrizio fractional derivative and application to groundwater pollution equation. Adv. Differ. Equ. 2016, 156 (2016)

16. Murio, D.A.: Implicit finite difference approximation for time fractional diffusion equations. Comput. Math. Appl. 56, $1138-1145(2008)$

17. Zheng, B., Gao, X., Yang, K., Zhang, C.: A novel meshless local Petrov-Galerkin method for dynamic coupled thermoelasticity analysis under thermal and mechanical shock loading. Eng. Anal. Bound. Elem. 60, 154-161 (2015)

18. Thamareerat, N., Luadsong, A., Aschariyaphotha, N.: Stability results of a fractional model for unsteady-state fluid flow problem. Adv. Differ. Equ. 2017, 74 (2017)

\section{Submit your manuscript to a SpringerOpen ${ }^{\circ}$ journal and benefit from:}

- Convenient online submission

- Rigorous peer review

- Open access: articles freely available online

- High visibility within the field

- Retaining the copyright to your article

Submit your next manuscript at $\downarrow$ springeropen.com 